\title{
Threshold Voltage Statistical Variability and Its Sensitivity to Critical Geometrical Parameters in Ultra-Scaled InGaAs and Silicon FETs
}

\author{
Nicolò Zagni, Francesco Maria Puglisi, Member, IEEE, Giovanni Verzellesi, Senior Member, IEEE, \\ and Paolo Pavan, Senior Member, IEEE
}

\begin{abstract}
We investigate the statistical variability of the threshold voltage and its sensitivity to critical geometrical parameters in Ultra-Scaled $I_{0.53} G_{0.47}$ As and Si MOSFETs by means of 3D quantum-corrected drift-diffusion simulations. Dual-Gate Ultra-Thin-Body and FinFET device structures are analyzed for both channel materials. To assess the variability and sensitivity effects also from the scaling perspective, we consider devices belonging to two technological nodes with gate lengths 15 $\mathrm{nm}$ and $10.4 \mathrm{~nm}$, designed according to ITRS specifications. Variability sources included in our analysis are Random Dopant Fluctuation (RDF), Work Function Fluctuation (WFF), as well as Body- and Gate- Line-Edge Roughness (LER). Sensitivity to critical geometrical parameters is assessed by varying gate length, channel thickness, and oxide thickness. Results point out the major detrimental effect of WFF and Body-LER for InGaAs FETs, whereas WFF dominates in Si counterparts. Moreover, the sensitivity analysis shows that control over gate length and channel thickness in InGaAs technology is fundamental in order to keep variability within tolerable values. Scaling of the InGaAs technology highlights the importance of abiding to ITRS projections regarding LER control improvement. Furthermore, a tight channel thickness control is required in ultra-scaled devices due to the large sensitivity of the threshold voltage to the channel thickness combined with increased variability.
\end{abstract}

Index Terms- III-V MOSFET, InGaAs, Line-Edge Roughness, Random Dopant Fluctuations, Sensitivity, Variability, Work-Function Fluctuations.

\section{INTRODUCTION}

$\mathrm{T}$ HE relentless scaling of CMOS technology is nowadays facing challenges in keeping up with Moore's law predictions. Among different alternatives to overcome the intrinsic limitations of Si-based devices, a very promising solution is replacing the thin Si channel with III-V materials [1]. Particularly, $\mathrm{In}_{0.53} \mathrm{Ga}_{0.47} \mathrm{As}$ is being extensively explored as a potential constituent of the channel in nMOS devices due

Manuscript received ??? March, 2017.

N. Zagni, F. M. Puglisi, and P. Pavan are with Dipartimento di Ingegneria "Enzo Ferrari", Università di Modena e Reggio Emilia, Via P. Vivarelli 10 int. 1, 41125, Modena (MO) - Italy (email: francescomaria.puglisi@unimore.it; phone: +39-059-2056324).

G. Verzellesi is with Dipartimento di Scienze e Metodi dell'Ingegneria, Università di Modena e Reggio Emilia, Via Amendola 2, 42122, Reggio Emilia (RE) - Italy.

(C) 2017 IEEE. Personal use of this material is permitted. Permission from IEEE must be obtained for all other uses, in any current or future media, including reprinting/republishing this material for advertising or promotional purposes, creating new collective works, for resale or redistribution to servers or lists, or reuse of any copyrighted component of this work in other works. to its superior electron mobility [2-4], which allows further dimension scaling, higher switching speed, and lower power consumption [1]. InGaAs superior mobility depends on the smaller electron effective mass that, however, has the side effect of enhancing quantization effects in the thin body and reducing the quantum capacitance (i.e., DOS bottleneck) [5, 6]. This makes InGaAs MOSFETs potentially more prone to both local and global variations of body thickness [7]. A capacitance and leads to stronger short-channel effects, which in turn can worsen the impact of gate-length fluctuations. In addition, substituting InGaAs for $\mathrm{Si}$ in the standard CMOS process flow poses major technological challenges to obtain the desired control on key process parameters (e.g., physical gate length $-\mathrm{L}_{\mathrm{G}}$, channel thickness - $\mathrm{T}_{\mathrm{CHAN}}$ ) [8]. In addition, the impact of technology scaling toward end-of-the-roadmap nodes deserves careful consideration, as $\mathrm{L}_{\mathrm{G}}$ and $\mathrm{T}_{\text {CHAN }}$ become comparable with the critical dimensions associated with intrinsic fluctuations (e.g., line-edge roughness amplitude and correlation length) [9]. All of these considerations call for an intensified effort towards the comprehension and the quantitative assessment of both statistical and systematic process variations in ultra-scaled InGaAs MOSFETs in view of their possible introduction into CMOS logic technology.

In this work, InGaAs FETs at the two technological nodes $\left(\mathrm{L}_{\mathrm{G}}=15 \mathrm{~nm}, \mathrm{~V}_{\mathrm{DD}}=0.63 \mathrm{~V}\right)$ and $\left(\mathrm{L}_{\mathrm{G}}=10.4 \mathrm{~nm}, \mathrm{~V}_{\mathrm{DD}}=0.59\right.$ V) are explored in terms of threshold voltage statistical variability and its sensitivity to systematic geometrical parameter variations, extending our recent conference report in [10]. Two types of device architecture are considered, namely the Dual-Gate Ultra-Thin-Body (DG-UTB) MOSFET and the FinFET. A comparison between InGaAs devices and their $\mathrm{Si}$ equivalent at the node $\left(\mathrm{L}_{\mathrm{G}}=15 \mathrm{~nm}, \mathrm{~V}_{\mathrm{DD}}=0.63 \mathrm{~V}\right)$ is also included for benchmarking purposes.

Variability is evaluated by using the statistical Impedance Field Method (IFM) [11] within the framework of quantum corrected drift-diffusion (QDD) three-dimensional device simulations. While unable to fully capture effects like mutual interactions among different variability sources as well as random location of discrete dopants and gate metal grains (which require more detailed atomistic simulations [12]), this smaller quantum capacitance reduces the overall gate 


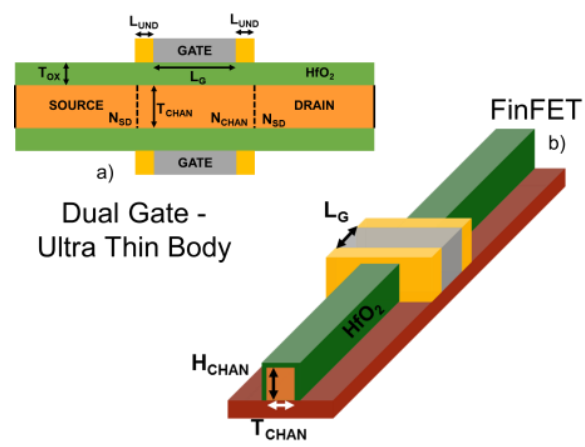

Fig. 1. Schematic representation of (a) Dual Gate Ultra Thin Body (DG-UTB) and (b) FinFET devices

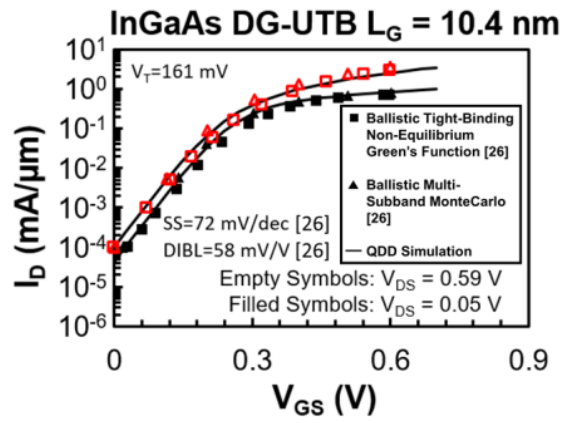

Fig. 4. Simulated $\mathrm{I}_{\mathrm{D}}-\mathrm{V}_{\mathrm{GS}}$ curves for the InGaAs DG-UTB device obtained using QDD (curves) and higher-order models (symbols) for $\mathrm{L}_{\mathrm{G}}=10.4 \mathrm{~nm}$.

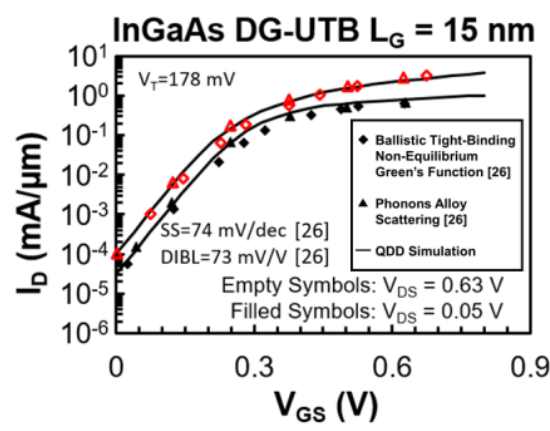

Fig. 2. Simulated $\mathrm{I}_{\mathrm{D}}-\mathrm{V}_{\mathrm{GS}}$ curves for the InGaAs DG-UTB device obtained using QDD (curves) and higher-order models (symbols) for $\mathrm{L}_{\mathrm{G}}=15$ $\mathrm{nm}$.

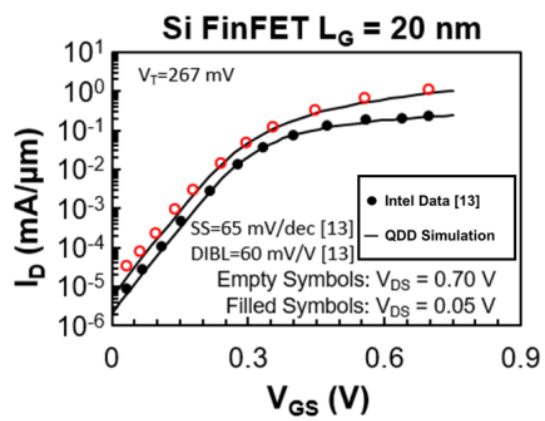

Fig. 5. Silicon FinFET QDD simulations (curves) calibrated over measurements data from Intel [13] (circles).

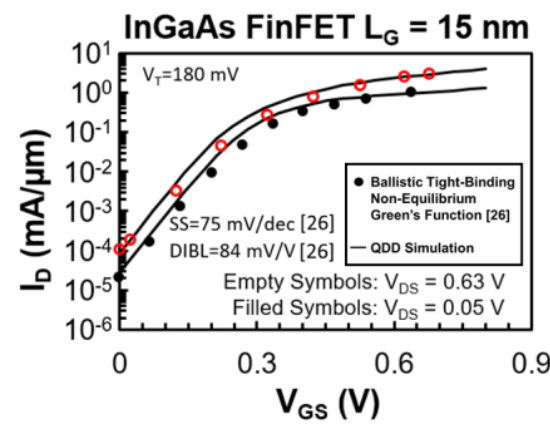

Fig. 3. Simulated $\mathrm{I}_{\mathrm{D}}-\mathrm{V}_{\mathrm{GS}}$ curves for the InGaAs FinFET device obtained using QDD (curves) and higher-order models (symbols) for $\mathrm{L}_{\mathrm{G}}=15 \mathrm{~nm}$.

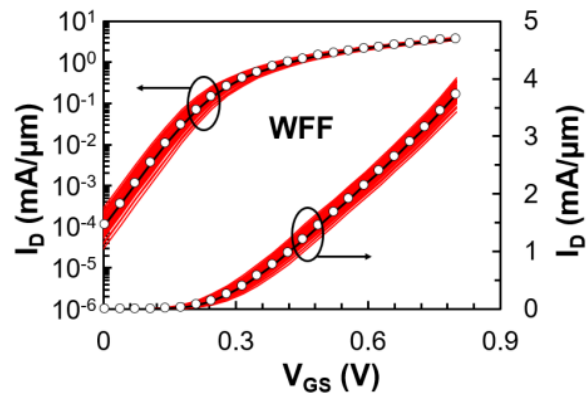

Fig. 6. Simulated $\mathrm{I}_{\mathrm{D}}-\mathrm{V}_{\mathrm{GS}}$ curves including variability (red curves, WFF effect on the InGaAs DG-UTB) over the ones of reference device (white circles).

TABLE I

GEOMETRICAL AND DOPING PARAMETERS OF REFERENCE DEVICES

\begin{tabular}{ccc}
\hline \hline Symbol & Quantity & Value \\
& Physical Gate Length & $15 \mathrm{~nm}-\mathbf{1 0 . 4} \mathbf{~ n m})$ \\
\hline $\mathrm{L}_{\mathrm{G}}$ & Gate Underlap & $2 \mathrm{~nm}$ \\
$\mathrm{~L}_{\mathrm{UND}}$ & Channel Thickness or Fin Width & $7 \mathrm{~nm}-4 \mathrm{~nm}$ \\
$\mathrm{~T}_{\mathrm{CHAN}}$ & Gate Width/Fin Height & $26 \mathrm{~nm}-14 \mathrm{~nm}$ \\
$\mathrm{~W}_{\mathrm{G}} / \mathrm{H}_{\mathrm{CHAN}}{ }^{\dagger}$ & Oxide Thickness & $3.84 \mathrm{~nm}^{*}-3.3 \mathrm{~nm}$ \\
$\mathrm{~T}_{\mathrm{OX}}$ & Channel Doping & $10^{17} \mathrm{~cm}^{-3}$ \\
$\mathrm{~N}_{\mathrm{CHAN}}$ & C
\end{tabular}

$*$ Value for DG-UTB devices. FinFETs have $\mathrm{T}_{\mathrm{OX}}=3.75 \mathrm{~nm}$.

$\dagger \mathrm{W}_{\mathrm{G}}$ refers to DG-UTB devices, $\mathrm{H}_{\mathrm{CHAN}}$ to FinFETs.

parameter variations, or simply sensitivity, respectively. In this Section is described the complete methodology used in our investigation, including analyzed device structures, calibration of simulations tools, and computational approaches adopted for the assessment of variability and sensitivity effects.

\section{A. Calibration}

The DG-UTB and FinFET structures for the two nodes (15 $\mathrm{nm}$ and $10.4 \mathrm{~nm}$ ) are implemented in the Synopsys Sentaurus Device $^{\mathrm{TM}}$ simulator according to ITRS projections, with geometrical and process parameters defined in Fig. 1 and Tab. I. These structures will be referred to as "reference devices" in the following. 3D numerical device simulations of these structures are carried out using the quantum-corrected driftdiffusion transport model (QDD) with quantum effects accounted for by means of the modified local density approximation (MLDA) model [25]. The calibration of the material-related properties (metal workfunction, effective channel electron mobility, saturation velocity) for InGaAs and
Ultra-scaled transistor technologies are susceptible to both 1) local fluctuations of dimensions/doping/material parameters ultimately related to discreteness of matter and charge, and 2) global variations of critical geometrical parameters and doping profiles induced by systematic process imperfections [15]. In the following, we will refer to effects 1) and 2) as statistical variability, or simply variability, and sensitivity to geometrical 
Si devices is performed separately. For all the investigated InGaAs devices (DG-UTB and FinFET at both nodes), it is possible to correctly reproduce the transfer characteristics in both linear and saturation regimes as obtained by higher-order transport models [26] with a unique set of QDD parameters, see Figs. 2-4 (where $\mathrm{V}_{\mathrm{T}}$ and short-channel parameters are also shown). Calibration for $\mathrm{Si}$ devices is performed by comparison with experimental data available in the open literature for a $\mathrm{Si}$ FinFET with $\mathrm{L}_{\mathrm{G}}=20 \mathrm{~nm}$ [13], see Fig. 5 (again including $\mathrm{V}_{\mathrm{T}}$ and short-channel parameters). The overall satisfactory agreement achieved for all materials and structures indicates that the adopted quantization model allows accurately describing the confinement effects in the ultra-thin body of these scaled devices.

\section{B. Variability}

Variability at the two technological nodes has been assessed by using the statistical Impedance Field Method (IFM) [11] available in Sentaurus Device ${ }^{\mathrm{TM}}$. Within the IFM, random device variations are treated as small perturbations of the nominal device. The full (3D) self-consistent device simulation is only performed once for each reference device, whereas current fluctuations at the device contacts can be calculated for a very large number of randomized realizations as the linear response to the applied device perturbations using a Green's function based approach. From the computed contact current fluctuations, a set of varied transfer curves can be constructed, from which the threshold voltage $\left(\mathrm{V}_{\mathrm{T}}\right)$ distribution is extracted. All the main microscopic variability sources of relevance for ultra-scaled MOSFETs [23] have been taken into account, namely: Random Dopant Fluctuations (RDF), Gate-metal Workfunction Fluctuations (WFF), Body-surface or Fin-Sidewall Roughness (BodyLER), and Gate Line Edge Roughness (Gate-LER). RDF, caused by inherent discreteness of impurity atoms, is modeled assuming spatially uncorrelated doping both in the body and in the source/drain regions. The number of dopant atoms in a given volume follows the Poisson distribution with an average value equal to the nominal number of dopants in that volume [27]. WFF is caused by intrinsic granularity of the gate metal, and modeled by assuming TiN gate metal characterized by two major grain orientations with a workfunction difference of $0.2 \mathrm{eV}$ and an associated probability of $0.4 / 0.6$ for the lowest/highest workfunction, respectively $[12,18]$. The number of metal grains in the gate region follows a Poisson distribution with an average value calculated using the average grain size. Each grain can have different shape and size, according to the procedure found in [27]. It is known that WFF variability increases with greater grain size, eventually saturating as grain size approaches the gate size itself [12]. Nonetheless, we consider an average grain size of $5 \mathrm{~nm}$ for both grain orientations as representative of the typical value achievable in present gate-first processes with $\mathrm{TiN}$ as the gate metal [28, 29]. Moreover, the grain random location also affects WFF variability; while our IFM-based approach is partially able to describe this effect, atomistic simulations are required for a more accurate analysis [12]. Line edge
TABLE II

PARAMETERS OF LINE EDGE ROUGHNESS VARIABILITY SOURCE

\begin{tabular}{cc}
\hline \hline $\begin{array}{c}\text { LER Parameters } \\
(\mathbf{1 5} \mathbf{~ n m})\end{array}$ & $\begin{array}{c}\text { LER Parameters } \\
(\mathbf{1 0 . 4} \mathbf{~ n m})\end{array}$ \\
\hline$\Delta_{\mathrm{rms}}=1.8 \mathrm{~nm}$ & $\Delta_{\mathrm{rms}}=1.2 \mathrm{~nm}$ \\
$\Lambda=15.5 \mathrm{~nm}$ & $\Lambda=8.3 \mathrm{~nm}$ \\
\hline \hline
\end{tabular}

roughness, inherent to patterning steps of CMOS technology, results in local variations of the gate length (Gate-LER) and of the body or fin thickness in DG-UTB and FinFET devices, respectively (Body-LER). These effects are modeled by means of a Gaussian autocorrelation function [9, 30], with r.m.s. roughness amplitude $\left(\Delta_{\mathrm{rms}}\right)$ and correlation length $(\Lambda)$ set in accordance with ITRS projections for the nodes under consideration [31]. For Gate-LER, the roughness at the two gate edges have been assumed uncorrelated, so that the combined, worst-case variability effects are taken into account. The same applies to roughness on the two body surfaces of the DG-UTB device or on the two fin sidewalls of the FinFET. The parameters used for WFF and Gate-/BodyLER are summarized in Tab. II (the model for RDF does not require any additional input parameter other than the doping profile). For each of the above variability sources, we consider 10000 randomly varied device realizations. $\mathrm{V}_{\mathrm{T}}$ is always evaluated at $\mathrm{V}_{\mathrm{DS}}=0.05 \mathrm{~V}$ with the constant current method at a reference current of $10 \mu \mathrm{A} / \mu \mathrm{m}$.

\section{Sensitivity}

Statistical variability is evaluated not only for the "reference devices" but also for varied devices resulting from the application of critical geometrical parameter variations, so that their interplay can be assessed. This is of paramount importance since classical analytical models of this interplay become inaccurate in ultra-scaled devices [14]. The effects of systematic variations are evaluated by applying uniform, global variations to the following parameters: gate length $\left(\mathrm{L}_{\mathrm{G}}\right)$, body thickness or fin width $\left(\mathrm{T}_{\mathrm{CHAN}}\right)$, and gate oxide thickness ( $\mathrm{T}_{\mathrm{ox}}$ ). Variations as large as $\pm 10 \%$ and $\pm 20 \%$ are applied to nominal values of these parameters. The variability analysis described in Section II-B is performed on all varied device realizations to extract the corresponding $\mathrm{V}_{\mathrm{T}}$ average and standard deviation values. In this paper, we purposely focus on $\mathrm{V}_{\mathrm{T}}$ variability/sensitivity effects, for which our modeling approach is best suited. The accurate estimation of the ONstate current variability would require higher-order transport models, accounting for quasi-ballistic transport and selfconsistent channel-mobility dependence on variability sources.

\section{RESULTS}

\section{A. Variability}

Bar plots in Fig. 7(a) provide a quantitative assessment of statistical variability at the $15-\mathrm{nm}$ node for both $\mathrm{Si}$ and InGaAs devices (DG-UTB and FinFET), showing extracted $\mathrm{V}_{\mathrm{T}}$ standard deviation value, $\sigma\left(\mathrm{V}_{\mathrm{T}}\right)$, for each variability source. The variability sources are considered separately from each other for the purpose of quantifying and comparing their individual contributions. Notably, only small differences 


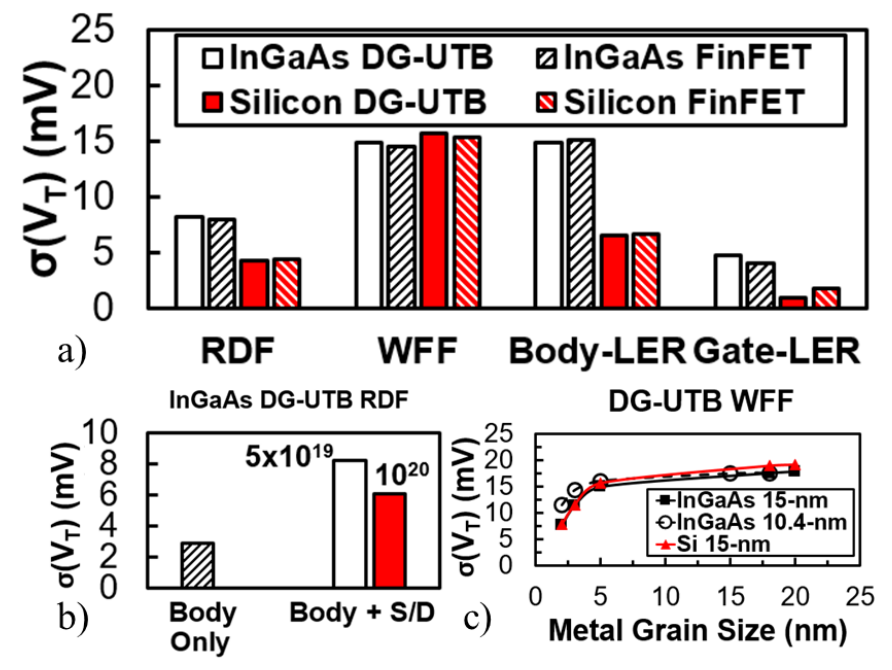

Fig. 7. (a) Bar plots of $\sigma\left(\mathrm{V}_{\mathrm{T}}\right)$ for the four variability sources and the four reference devices at the 15-nm node. (b) RDF variability for the InGaAs DGUTB device, when considering body doping variations only and both body and S/D doping variations, for two different S/D doping concentrations, namely $5 \times 10^{19} \mathrm{~cm}^{-3}$ (white bin) and $10^{20} \mathrm{~cm}^{-3}$ (red bin). (c) WFF variability for the DG-UTB devices, with varying metal grain size for the InGaAs (15$\mathrm{nm}$ and 10.4-nm node) and Si technology.

between DG-UTB and FinFET architectures arise, regardless of the considered variability source. It is evident from Fig. 7 (a) that InGaAs devices show higher Body-LER and GateLER as well as RDF variability than Si FETs. This can be understood by considering that InGaAs has a smaller effective mass than $\mathrm{Si}$, resulting in: $i$ ) larger quantum confinement effects, making $\mathrm{V}_{\mathrm{T}}$ more susceptible to channel thickness fluctuations (larger Body-LER variability) [32]; ii) reduced effective inversion capacitance, that makes $\mathrm{V}_{\mathrm{T}}$ more sensitive to modulation of gate length and associated short-channel effects (larger Gate-LER variability). Interestingly, RDF is affected by the lower InGaAs density of states, leading to a non-negligible dependence of $\mathrm{V}_{\mathrm{T}}$ on source/drain doping concentration (i.e., "source-starvation effect" [6]). This can be appreciated in Fig. $7(\mathrm{~b})$, where $\sigma\left(\mathrm{V}_{\mathrm{T}}\right)$ is shown for the InGaAs DG-UTB device, with RDF activated in the body region only and in the entire device (body + S/D) for two different $\mathrm{N}_{\mathrm{S} / \mathrm{D}}$ values (namely $\mathrm{N}_{\mathrm{S} / \mathrm{D}}=5 \times 10^{19}, 10^{20} \mathrm{~cm}^{-3}$ ). Remarkably, $\sigma\left(\mathrm{V}_{\mathrm{T}}\right)$ decreases at increasing $\mathrm{N}_{\mathrm{S} / \mathrm{D}}$, and it is minimum when $\mathrm{RDF}$ is accounted for in the body region only.

Figure 7(c) shows the effect of different metal grain size (in the range of 2 to $20 \mathrm{~nm}[33,34])$ on the WFF variability for the DG-UTB devices. Results show that for grain size $>5 \mathrm{~nm}$ $\sigma\left(V_{T}\right)$ only slightly increases. In addition, specific works in the literature focusing on the WFF variability show that, by adopting a more realistic tessellation of the gate area, the forecasted increase of WFF variability at grain size $>5 \mathrm{~nm}$ is further reduced, see [34]. On the other hand, grain size $<5 \mathrm{~nm}$ causes WFF variability to drop considerably. Nevertheless, this reduction can only be achieved with processes preventing the crystallization of the metal [35], i.e., gate-last flow, or with different materials, such as TaSiN, that remain amorphous even in gate-first flows [36]. Hereafter, we consider $\sigma\left(\mathrm{V}_{\mathrm{T}}\right)$ at $5 \mathrm{~nm}$ grain size to be representative of WFF variability for the typical gate-first processes in the devices under study, with

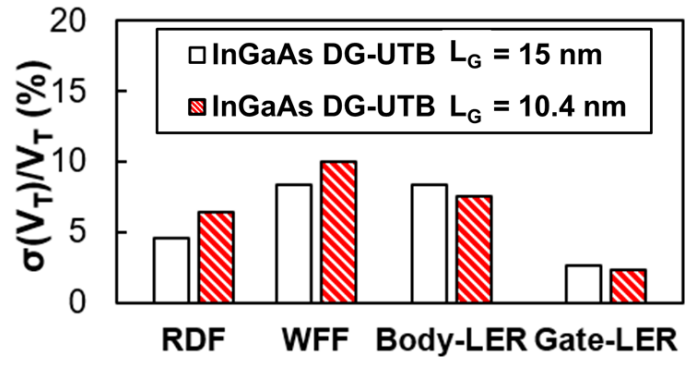

Fig. 8. Bar plots of $\sigma\left(\mathrm{V}_{\mathrm{T}}\right) / \mathrm{V}_{\mathrm{T}}$ for the four variability sources and the two InGaAs DG-UTB reference devices at two technological nodes.

TiN as the gate metal. In general, WFF has comparable effects on InGaAs and Si devices due to the same device geometries. Another important point about $\sigma\left(\mathrm{V}_{\mathrm{T}}\right)$ data shown in Fig. $7(\mathrm{a})$ is that WFF and Body-LER have comparable and predominant variability effects on $V_{T}$ of InGaAs devices, whereas WFF is the only predominant source of variability in Si.

Bar plots in Fig. 8 provide a quantitative comparison of statistical variability at the $15-\mathrm{nm}$ and $10.4-\mathrm{nm}$ nodes, showing the extracted $\sigma\left(\mathrm{V}_{\mathrm{T}}\right)$ values for the different variability sources, similarly to what reported in Fig. 7(a). Note that, for the sake of comparison, standard deviation values are normalized to corresponding average values $\mu\left(V_{T}\right)$. Notably, the relative variability of $\mathrm{V}_{\mathrm{T}}$ is not dramatically affected by $\mathrm{L}_{\mathrm{G}}$ scaling. More specifically, i) RDF- and WFF-induced variability slightly increases at the 10.4-nm node mostly due to the small nominal $\mathrm{V}_{\mathrm{T}}$ reduction; ii) Body-LER and GateLER variability actually decreases with $\mathrm{L}_{\mathrm{G}}$ scaling due to the reduction in both $\Delta_{\mathrm{rms}}$ and $\Lambda$ parameters projected by ITRS and employed in our simulations (see Tab. II). In this scenario, the detrimental impact of Body-LER on $\mathrm{V}_{\mathrm{T}}$ variability evident for the 15-nm node is attenuated, leaving to WFF the role of dominant variability source (as for Si devices). However, this encouraging scaling perspective holds true only if the LER control improvements forecasted by ITRS are effectively attained.

\section{B. Sensitivity}

The overall promising picture emerging from the variability analysis needs to be confirmed also when considering the effects of systematic geometrical parameter variations. For this purpose, we have considered all the varied and reference devices and evaluated how $\mu\left(\mathrm{V}_{\mathrm{T}}\right)$ and $\sigma\left(\mathrm{V}_{\mathrm{T}}\right)$ are affected by systematic variations. We focus the analysis only on DG-UTB devices, as they exhibit comparable variability with respect to their FinFET counterparts, as reported in Fig. 7(a). Results are reported in Fig. 9 for the InGaAs DG-UTB devices at the 15$\mathrm{nm}$ and 10.4-nm nodes as well as for the Si DG-UTB device at the 15-nm node. Figures 9(a)-9(c) show the dependence of

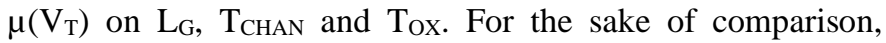
$\mu\left(V_{T}\right)$ values are normalized to the $V_{T}$ of the reference device.

As it can be noted, the 15-nm node InGaAs device exhibits worse or, at most, similar $\mathrm{V}_{\mathrm{T}}$ sensitivity than the Si FET, for all varied parameters. This is still a consequence of the reduced quantum capacitance of the InGaAs MOSFET making the device more prone to short-channel effects and therefore translating to: 

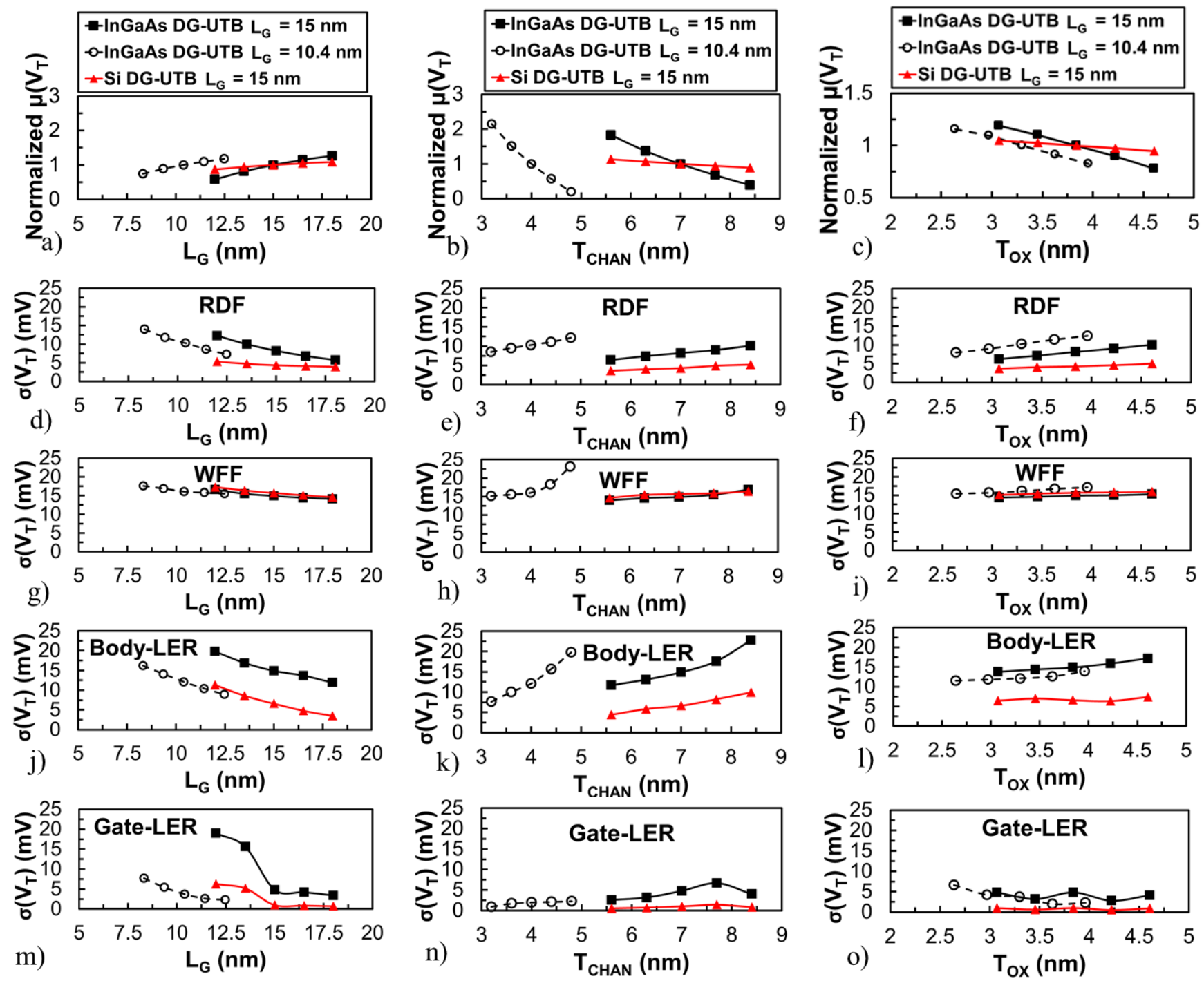

Fig. 9. Plots of the sensitivity of $\mu\left(V_{T}\right)$ normalized to the $V_{T}$ of the reference device to (a) $L_{G}$, (b) $T_{C H A N}$, and (c) $T_{\text {OX }}$. Plots of $\sigma\left(V_{T}\right)$ sensitivity to (d-g-j-m) $L_{G}$, (e-h-k-n) $\mathrm{T}_{\text {CHAN }}$, and (f-i-l-o) $\mathrm{T}_{\mathrm{OX}}$ due to (d-e-f) RDF, (g-h-i) WFF, (j-k-1) Body-LER, and (m-n-o) Gate-LER. Filled (empty) black symbols refer to InGaAs 15nm (10.4-nm) node DG-UTB devices. Red symbols refer to Si DG-UTB devices.

1) a more significant $V_{T}$ roll-off with $L_{G}$ decrease, Fig. 9(a);

2) a larger $\mathrm{V}_{\mathrm{T}}$ increase with shrinking of $\mathrm{T}_{\mathrm{CHAN}}$, see Fig. 9(b), this being the predominant variation effect in both the InGaAs and the Si device;

3) a larger $V_{T}$ increase upon decreasing in $T_{O X}$, see Fig. 9 (c). It is worth noticing that an increase in $V_{T}$ at decreasing $\mathrm{T}_{\mathrm{OX}}$ is indeed opposite to what generally expected and can be ascribed to strong quantization effects in these ultra-narrow channel devices [37].

The observed larger $\mu\left(V_{\mathrm{T}}\right)$ sensitivity to geometrical parameter variations in InGaAs devices, as compared to $\mathrm{Si}$ ones, at the $15-\mathrm{nm}$ node raises concerns about the scaling potential of InGaAs devices. Our analysis show that node scaling weakly affects $\mu\left(V_{T}\right)$ sensitivity to variation of critical geometrical parameters, as far as $\mathrm{L}_{\mathrm{G}}$ and $\mathrm{T}_{\mathrm{OX}}$ are concerned. This can be appreciated in Fig. 9(a) and 9(c) showing that the $\mu\left(V_{T}\right)$ curves versus $L_{G}$ and $T_{O X}$ almost rigidly shift to smaller dimensions upon scaling. $\mathrm{T}_{\text {CHAN }}$ scaling causes instead more concern, as the $\mu\left(\mathrm{V}_{\mathrm{T}}\right)$ roll-off at increasing $\mathrm{T}_{\mathrm{CHAN}}$ becomes steeper upon node scaling, see Fig. 9(b). In this case, for a
$20 \%$ increase above the nominal value (i.e., a $0.8 \mathrm{~nm}$ change), $\mu\left(\mathrm{V}_{\mathrm{T}}\right)$ drops below $50 \mathrm{mV}$. The sensitivity of $\sigma\left(\mathrm{V}_{\mathrm{T}}\right)$ on systematic geometrical parameter variations is illustrated in Figs. 9(d)-9(o), for the different statistical variability sources. InGaAs and Si DG-UTB MOSFETs are again compared. As can be noted, qualitatively similar $\sigma\left(\mathrm{V}_{\mathrm{T}}\right)$ dependence on varying parameters are predicted for the two materials at the 15-nm node. However, similarly to what is observed for the nominal structures (see Fig. 7(a)), the InGaAs devices always display worse or, at most, comparable $\sigma\left(\mathrm{V}_{\mathrm{T}}\right)$ than $\mathrm{Si}$ ones, regardless of the varying parameter and the statistical variability source considered. This holds true also for the FinFET structure [10]. Most of the $\sigma\left(\mathrm{V}_{\mathrm{T}}\right)$-vs-parameter trends shown in Figs. 9(d)-9(o) actually confirm results for ultra-thinchannel devices, already pointed out by studies on $\mathrm{Si}$ MOSFETs [9], indicating that: i) WFF variability has very small dependence on geometrical systematic variations; ii) statistical $\mathrm{V}_{\mathrm{T}}$ variability worsens at decreasing $\mathrm{L}_{\mathrm{G}}$ as well as increasing $\mathrm{T}_{\mathrm{CHAN}}$ and $\mathrm{T}_{\mathrm{OX}}$ for RDF, Body-, and Gate-LER. The latter is the most crucial aspect, as it emphasizes the 


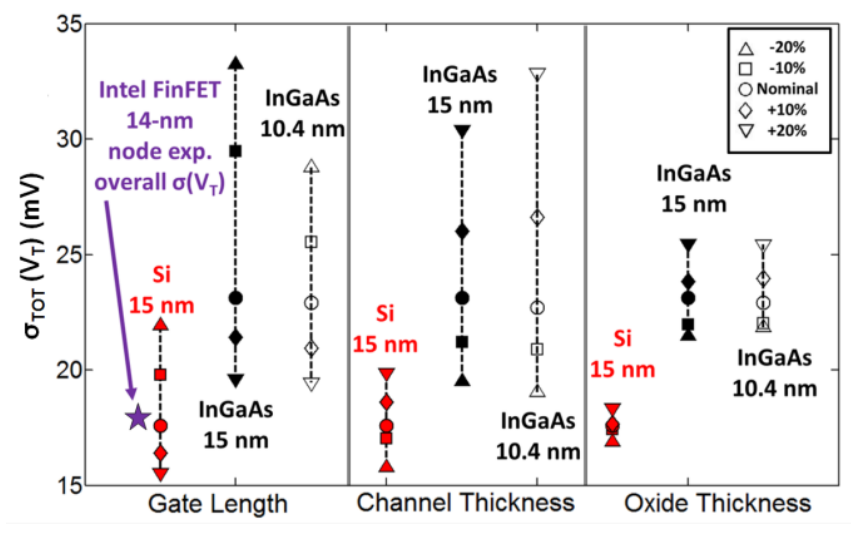

Fig. 10. Sensitivity of the overall variability $\sigma_{\mathrm{TOT}}\left(\mathrm{V}_{\mathrm{T}}\right)$ (i.e., sum of all variability sources) to $\mathrm{L}_{\mathrm{G}}, \mathrm{T}_{\mathrm{CHAN}}$ and $\mathrm{T}_{\mathrm{OX}}$ for the $15-\mathrm{nm}$ node $\mathrm{Si}$ (red), $15-\mathrm{nm}$ and 10.4-nm node InGaAs DG-UTB devices (black filled and hollow symbols, resepctively). Experimental data is included for the Si technology (purple star) for the 14-nm node $\left(\mathrm{L}_{\mathrm{G}}=20 \mathrm{~nm}\right)$.

importance of appropriate scaling and accurate control of involved critical geometrical parameters. In this context, the sensitivity of $\sigma\left(\mathrm{V}_{\mathrm{T}}\right)$ to geometrical parameter variations in InGaAs devices is, in most cases, not appreciably worsened by scaling.

A notable exception to this picture is evidenced in Fig. 9(h) for the 10.4-nm node InGaAs device, which displays a worsening of the $\sigma\left(\mathrm{V}_{\mathrm{T}}\right)$ sensitivity to $\mathrm{T}_{\mathrm{CHAN}}$ due to WFF. This $\sigma\left(\mathrm{V}_{\mathrm{T}}\right)$ increase for increasing $\mathrm{T}_{\mathrm{CHAN}}$, combined with corresponding $\mu\left(V_{T}\right)$ decrease observed in Fig. 9(b) may be a critical issue for device scaling. In any case, scaling to the 10.4-nm node results in an increased sensitivity of $\sigma\left(\mathrm{V}_{\mathrm{T}}\right)$ to $\mathrm{T}_{\text {CHAN }}$ also for Body-LER variability (which is the dominant variability source along with WFF). This indicates that, despite ITRS projections on LER control are followed when scaling to the 10.4-nm node (see Tab. II), the $\sigma\left(\mathrm{V}_{\mathrm{T}}\right)$ sensitivity to $\mathrm{T}_{\mathrm{CHAN}}$ worsens. Eventually, the combination of the increased sensitivity to $\mathrm{T}_{\mathrm{CHAN}}$ for both $\mu\left(\mathrm{V}_{\mathrm{T}}\right)$ and $\sigma\left(\mathrm{V}_{\mathrm{T}}\right)$ can be a showstopper for this technology, highlighting the critical role of $\mathrm{T}_{\text {CHAN }}$ control, which is discussed in the next Section.

\section{DISCUSSION}

To assess the overall tolerance of a technology to process variations, it is necessary to evaluate the total $\mathrm{V}_{\mathrm{T}}$ variability by considering the combined effect of all variability sources. Here we assume sources to be mutually independent, thus evaluating the total variability, $\sigma_{\mathrm{TOT}}\left(\mathrm{V}_{\mathrm{T}}\right)$, by using equation (2) in [15]. A comparison between the $\sigma_{\text {Tот }}\left(\mathrm{V}_{\mathrm{T}}\right)$ of InGaAs and Si DG-UTB devices is reported in Fig. 10, along with its dependence on $\mathrm{L}_{\mathrm{G}}, \mathrm{T}_{\mathrm{CHAN}}$, and $\mathrm{T}_{\mathrm{OX}}$ variations. Remarkably, our results are in good agreement with recent experimental data showing a $\sigma_{\text {тот }}\left(\mathrm{V}_{\mathrm{T}}\right)$ of about $18 \mathrm{mV}$ for Si FinFETs at the 14-nm node [13] (purple star symbol in Fig. 10).

Interactions among different variability effects can in principle result in either over- or under-estimation of total variability, and their evaluation requires atomistic approaches to be adopted [12]. In [38], authors investigate InGaAs-based FinFETs and show that considering the LER/WFF coupling can result in a larger variability, as compared to the case in

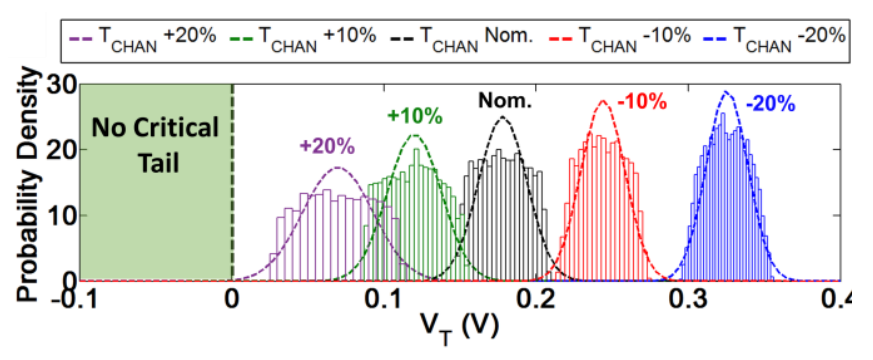

Fig. 11. Sensitivity of $\mathrm{V}_{\mathrm{T}}$ distributions obtained from the sum of all variability sources to $T_{\text {CHAN }}$ for the 15 -nm node InGaAs DG-UTB device.

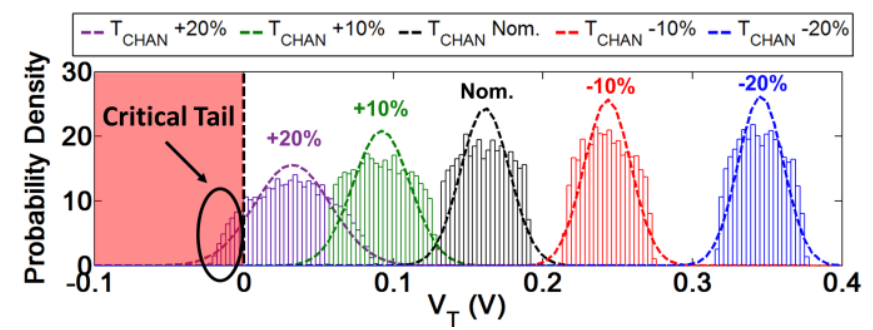

Fig. 12. Sensitivity of $\mathrm{V}_{\mathrm{T}}$ distributions obtained from the sum of all variability sources to $\mathrm{T}_{\text {CHAN }}$ for the 10.4 -nm node InGaAs DG-UTB device. An increas of $\mathrm{T}_{\text {CHAN }}$ might lead to a significant chance of having normally-on devices.

which the coupling is neglected. However, the effect is nonnegligible only when the two sources are independently characterized by comparable $\sigma$ values. Since this could be the case of InGaAs 15-nm devices, see Fig. 7(a), the total variability could be worse than the predictions in Fig. 10. Conversely, in the case in which one source is dominant, the coupling effect can be safely ignored. On the other hand, RDF and LER components have been suggested to be statistically independent $[12,39]$. Overall, our analysis leads to reasonable values, as demonstrated by the comparison with experimental results for $\mathrm{Si}$, which improves our confidence on the dependability of our variability predictions also for InGaAs devices, for which experimental data is not yet available on statistically meaningful data sets.

Regardless of the geometrical parameter under consideration, InGaAs devices exhibit higher $\sigma_{\mathrm{TOT}}\left(\mathrm{V}_{\mathrm{T}}\right)$ than the $\mathrm{Si}$ equivalent at the $15-\mathrm{nm}$ node, and show a larger $\sigma_{\text {Tот }}\left(\mathrm{V}_{\mathrm{T}}\right)$ dependence on all parameters. Interestingly, scaling InGaAs devices to the 10.4-nm node negligibly affects their overall variability and also results in significantly better $\sigma_{\mathrm{TOT}}\left(\mathrm{V}_{\mathrm{T}}\right)$ sensitivity to all parameters, with the exception of $\mathrm{T}_{\text {CHAN. The critical role of }} \mathrm{T}_{\text {CHAN }}$ control upon scaling is further illustrated in Figs. 11 and 12, showing the $\mathrm{V}_{\mathrm{T}}$ distributions of InGaAs DG-UTB devices for the different

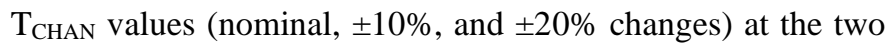
technological nodes, respectively. Specifically, a $\mathrm{T}_{\text {CHAN }}$ increase can be critical due to the IOFF increase associated with $\mathrm{V}_{\mathrm{T}}$ reduction, a scenario which is further worsened by the associated $\sigma_{\mathrm{TOT}}\left(\mathrm{V}_{\mathrm{T}}\right)$ increase (standard deviation of the distributions in Figs. 11 and 12). In particular, our results show that, at the $15-\mathrm{nm}$ node, a $10 \% \mathrm{~T}_{\mathrm{CHAN}}$ increase results in an almost $30 \%$ probability to obtain a device with $\mathrm{V}_{\mathrm{T}}<100$ $\mathrm{mV}$ (see Fig. 11), which implies a $>10 \mathrm{x}$ increase in IOFF. This picture is exacerbated at the 10.4-nm node: a $\mathrm{T}_{\mathrm{CHAN}}$ increase of $10 \%$ results in almost $30 \%$ chance to get a device with $\mathrm{V}_{\mathrm{T}}$ 
$<70 \mathrm{mV}$ (see Fig. 12), which implies a >50x increase in IOFF. At this node, a further $\mathrm{T}_{\text {CHAN }}$ increase (i.e., $+20 \%$ ) might even lead to a significant chance to get a normally-on device (critical tail highlighted in Fig. 12). On the other hand, a $\mathrm{T}_{\text {CHAN }}$ decrease results in obvious gate overdrive and $\mathrm{I}_{\mathrm{ON}}$ current reduction. Particularly, for both technological nodes a $\approx 20 \%$ reduction in $\mathrm{I}_{\mathrm{ON}}$ is observed for a $10 \% \mathrm{~T}_{\mathrm{CHAN}}$ reduction. Likewise, the observed $\mathrm{I}_{\mathrm{ON}}$ decrease is larger than $40 \%$ considering a $20 \% \mathrm{~T}_{\mathrm{CHAN}}$ reduction. Therefore, $\mathrm{T}_{\mathrm{CHAN}}$ control appears to be the most stringent constraint for InGaAs technology when variability/sensitivity effects are considered. Approaches that rely on $\mathrm{T}_{\mathrm{CHAN}}$ definition by epitaxy rather than etching, like in [40] and [41] for UTB and FinFET devices, respectively, might represent a viable solution to reduce $\mathrm{T}_{\text {CHAN }}$ sensitivity as well as related LER effects.

\section{CONCLUSIONS}

In this work, we investigated the effects of both statistical variability and systematic geometrical parameter variations on InGaAs DG-UTB and FinFET devices at two technological nodes $\left(\mathrm{L}_{\mathrm{G}}=15 \mathrm{~nm}, \mathrm{~V}_{\mathrm{DD}}=0.63 \mathrm{~V}\right)$ and $\left(\mathrm{L}_{\mathrm{G}}=10.4 \mathrm{~nm}, \mathrm{~V}_{\mathrm{DD}}=\right.$ $0.59 \mathrm{~V})$. For the $15 \mathrm{~nm}$-node, the analysis has been extended to Si-based devices for benchmarking purposes. Our simulations show that InGaAs devices have comparatively higher $V_{T}$ variability than $\mathrm{Si}$ ones as a result of stronger quantization effects in the ultra-thin device channel. Moreover, InGaAs devices exhibit worse $\mathrm{V}_{\mathrm{T}}$ sensitivity to systematic variations. Nevertheless, the overall variability, due to the combined effects of all variability sources, appears still manageable at the 15 -nm node (i.e., smaller than $30 \mathrm{mV}$ considering $\pm 10 \%$ deviation on $\mathrm{L}_{\mathrm{G}}$ or $\left.\mathrm{T}_{\mathrm{CHAN}}\right)$. Scaling to the 10.4-nm node negligibly affects $\mathrm{V}_{\mathrm{T}}$ variability, if ITRS projections regarding LER control are attained. On the other hand, scaling strongly affects $V_{\mathrm{T}}$ sensitivity to $\mathrm{T}_{\mathrm{CHAN}}$, thus configuring the control over $\mathrm{T}_{\mathrm{CHAN}}$ as the most critical issue for InGaAs devices scaling.

\section{ACKNOWLEDGMENT}

This research has received funding from the European Commission's Seventh Framework Programme (FP7/20072013) under grant agreement III-V-MOS Project $n^{\circ} 619326$.

\section{REFERENCES}

[1] J. A. del Alamo, "Nanometre-scale electronics with III-V compound semiconductors," Nature, vol. 479, pp. 317-323. [Online] Available: http://www.nature.com/nature/journal/v479/n7373/full/nature10677.html

[2] P. Pavan, N. Zagni, F. M. Puglisi, A. Alian, A. V.-Y. Thean, N. Collaert, and G. Verzellesi, "The impact of interface and border traps on currentvoltage, capacitance-voltage, and split-CV mobility measurements in InGaAs MOSFETs," Phys. Status Solidi A, vol. 214, no. 3, pp. 16005921600599, Mar. 2017. DOI: 10.1002/pssa.201600592.

[3] A. Alian, M. A. Pourghaderi, Y. Mols, M. Cantoro, T. Ivanov, N. Collaert, and A. Thean, "Impact of the channel thickness on the performance of ultrathin InGaAs channel MOSFET devices," in Proc. IEEE Inter. Electron Devices Meeting (IEDM), San Francisco, CA, USA, 2013, pp. 16.6.1-16.6.4. DOI: 10.1109/IEDM.2013.6724644.

[4] L. Morassi, G. Verzellesi, H. Zhao, J. C. Lee, D. Veksler, and G. Bersuker, "Errors Limiting Split-CV Mobility Extraction Accuracy in Buried-Channel InGaAs MOSFETs," IEEE Trans. Electron Devices, vol. 59, no. 4, pp. 1068-1075, Apr. 2012. DOI: 10.1109/TED.2011.2182513.

[5] S. Takagi, T. Iisawa, T. Tesuka, T. Numata, S. Nakaharai, N. Hirashita, Y. Moriyama, K. Usuda, E. Toyoda, S. Dissanayake, M. Shichijo, R. Nakane, S. Sugahara, M. Takenaka, and N. Sugiyama, "CarrierTransport-Enhanced Channel CMOS for Improved Power Consumption and Performance," IEEE Trans. Electron Devices, vol. 55, no. 1, pp. 2139, Jan. 2008. DOI: 10.1109/TED.2007.911034.

[6] N. Neophytou, T. Rakshit and M. S. Lundstrom, "Performance Analysis of 60-nm Gate-Length III-V InGaAs HEMTs: Simulations Versus Experiments," IEEE Trans. Electron Devices, vol. 56, no. 7, pp. 13771387, Jul. 2009. DOI: 10.1109/TED.2009.2021437.

[7] A. Nidhi, V. Saripalli, V. Narayanan, Y. Kimura, R. Arghavani, and S. Datta, "Will strong quantum confinement effect limit low VCC logic application of III-V FINFETs?," in Proc. 70 ${ }^{\text {th }}$ Device Research Conference (DRC), University Park, TX, 2012, pp. 231-232. DOI: 10.1109/DRC.2012.6256968.

[8] R. J. W. Hill, C. Park, J. Barnett, J. Price, J, Huang, N. Goel, W. Y. Loh, J. Oh, C. E. Smith, P. Kirsch, P. Majhi, and R. Jammy, "Self-aligned III$\mathrm{V}$ MOSFETs heterointegrated on a $200 \mathrm{~mm} \mathrm{Si}$ substrate using an industry standard process flow," in IEEE Inter. Electron Devices Meeting, San Francisco, CA, USA, 2010, pp. 6.2.1-6.2.4. DOI: 10.1109/IEDM.2010.5703307.

[9] A. Asenov, S. Kaya, and A. R. Brown, "Intrinsic parameter fluctuations in decananometer MOSFETs introduced by gate line edge roughness", IEEE Trans. Electron Devices, vol. 50, no. 5, pp. 1254-1260, May 2003. DOI: 10.1109/TED.2003.813457

[10] N. Zagni, F. M. Puglisi, G. Verzellesi, and P. Pavan, "Combined Variability/Sensitivity Analysis in III-V and Silicon FETs for Future Technological Nodes," in Proc. IEEE Int. Rel. Phys. Symp. (IRPS), Monterey, CA, USA, Apr. 2-6, 2017.

[11] K. El Sayed, E. Lyumkis, and A. Wettstein, "Modeling statistical variability with the impedance field method," in Proc. of SISPAD, pp. 205-208, Sept. 2012. ISBN: 978-0-615-71756-2.

[12] A. R. Brown, N. M. Idris, J. R. Watling, and A. Asenov, "Impact of Metal Gate Granularity on Threshold Voltage Variability: A Full-Scale Three-Dimensional Statistical Simulation Study," IEEE Electron Device Letters, vol. 31, no. 11, pp. 1199-1201, Nov. 2010. DOI: 10.1109/LED.2010.2069080.

[13] S. Natarajan, M. Agostinelli, S. Akbar, M. Bost, A. Bowonder, V. Chikarmane, S. Chouksey, A. Dasgupta, K. Fischer, Q. Fu, T. Ghani, M. Giles, S. Govindaraju, R. Grover, W. Han, D. Hanken, E. Haralson, M. Haran, M. Heckscher, R. Heussner, P. Jain, R. James, R. Jhaveri, I. Jin, H. Kam, E. Karl, C. Kenyon, M. Liu, Y. Luo, R. Mehandru, S. Morarka, L. Neiberg, P. Packan, A. Paliwal, C. Parker, P. Patel, R. Patel, C. Pelto, L. Pipes, P. Plekhanov, M. Prince, S. Rajamani, J. Sandford, B. Sell, S. Sivakumar, P. Smith, B. Song, K. Tone, T. Troeger, J. Wiedemer, M. Yang, and K. Zhang, "A $14 \mathrm{~nm}$ logic technology featuring $2^{\text {nd }}$-generation FinFET, air-gapped interconnects, self-aligned double patterning and a $0.0588 \mu \mathrm{m}^{2}$ SRAM cell size," in Proc. IEEE Inter. Electron Devices Meeting (IEDM), San Francisco, CA, USA, 2014, pp. 3.7.1-3.7.3. DOI: 10.1109/IEDM.2014.7046976.

[14] X. Wang, B. Cheng, A. R. Brown, C. Millar, J. B. Kuang, S. Nassif, and A. Asenov, "Interplay between process-induced and statistical variability in 14-nm CMOS technology double-gate SOI FinFETs," IEEE Trans. Electron Devices, vol. 60, no. 8, pp. 2485-2492, Aug. 2013. DOI: 10.1109/TED.2013.2267745.

[15] Y. Li, C.-H. Hwang, T.-Y. Li, and M.-H. Han, "Process-variation effect, metal-gate workfunction fluctuation, and random-dopant fluctuation in emerging CMOS technologies," IEEE Trans. Electron Devices, vol. 57, no. 2, pp. 437-447, Feb. 2010. DOI: 10.1109/TED.2009.2036309.

[16] X. Wang, F. Adamu-Lema, B. Cheng, and A. Asenov, "Geometry, temperature, and body bias dependence of statistical variability in 20-nm bulk CMOS technology: a comprehensive simulation analysis," IEEE Trans. Electron Devices, vol. 60, no. 5, pp. 1547-1554, May 2013. DOI: 10.1109/TED.2013.2254490.

[17] G. Leung, and C. O. Chui, "Interactions between line edge roughness and random dopant fluctuation in nonplanar field-effect transistor variability," IEEE Trans. Electron Devices, vol. 60, no. 10, pp. 32773284, Oct. 2013. DOI: 10.1109/TED.2013.2276072.

[18] L. Gerrer, A. R. Brown, C. Millar, R. Hussin, S. M. Amoroso, B. Cheng, D. Reid, C. Alexander, D. Fried, M. Hargrove, K. Greiner, A. Asenov, 
"Accurate Simulation of Transistor-Level Variability for the Purposes of TCAD-Based Device-Technology Cooptimization," in IEEE Trans. Electron Devices, vol. 62, no. 6, pp. 1739-1745, Jun. 2015. DOI: 10.1109/TED.2015.2402440.

[19] M. Wong, K. D. Holland, S. Anderson, S. Rizwan, Z. C. J. Yuan, T. B. Hook, D. Kienle, P. S. Gudem, and M. Vaidyanathan, "Impact of ShortWavelength and Long-Wavelength Line-Edge Roughness on the Variability of Ultrascaled FinFETs," IEEE Trans. Electron Devices, vol. 64, no. 3, pp. 1231-1238, Mar. 2017. DOI: 10.1109/TED.2017.2655520.

[20] Y. Lee, and C. Shin, "Impact of Equivalent Oxide Thickness on Threshold Voltage Variation Induced by Work-Function Variation in Multigate Devices," IEEE Trans. Electron Devices, vol. 64, no. 5, pp. 2452-2456, May 2017. DOI: 10.1109/TED.2017.2673859.

[21] N. Agrawal, Y. Kimura, R. Arghavani, and S. Datta, "Impact of transistor architecture (bulk planar, trigate on bulk, ultrathin-body planar SOI) and material (Silicon or III-V semiconductor) on variation for logic and SRAM applications," IEEE Trans. Electron Devices, vol. 60, no. 10, pp. 3298-1697-3304, Aug. 2013. DOI: 10.1109/TED.2013.2277872.

[22] G. Leung, A. Pan, and C. O. Chui, "Junctionless Silicon and $\mathrm{In}_{0.53} \mathrm{Ga}_{0.47}$ As Transistors-Part II: Device Variability From Random Dopant Fluctuation," IEEE Trans. Electron Devices, vol. 62, no. 10, pp. 3208-3214, Oct. 2015, 10.1109/TED.2015.2464298.

[23] N. Agrawal, H. Liu, R. Arghavani, V. Narayanan, and S. Datta, "Impact of variation in nanoscale Silicon and non-Silicon FinFETs and tunnel FETs on device and SRAM variability," IEEE Trans. Electron Devices, vol. 62, no. 6, pp. 1691-1697, Jun. 2015. DOI: 10.1109/TED.2015.2406333.

[24] N. Seoane, G. Indalecio, M. Aldegunde, D. Nagy, M. A. Elmessary, A. J. García-Loureiro, and K. Kalna, "Comparison of Fin-Edge Roughness and Metal Grain Work Function Variability in InGaAs and Si FinFETs," IEEE Trans. Electron Devices, vol. 63, no. 3, pp. 1209-1216, Mar. 2016. DOI: 10.1109/TED.2016.2516921.

[25] G. Paasch, and H. Übensee, "A Modified Local Density Approximation. Electron Density in Inversion Layers," Physica Status Solidi B, vol. 113, no. 1, pp. 165/178, Sep. 1982. DOI: 10.1002/pssb.2221130116

[26] M. Rau, E. Caruso, D. Lizzit, P. Palestri, D. Esseni, A. Schenk, L. Selmi, and M. Luisier, "Performance Projection of III-V Ultra-Thin-Body, FinFET, and Nanowire MOSFETs for two Next-Generation Technology Nodes," in IEEE Inter. Electron Devices Meeting, San Francisco, CA, 2016, pp. 30.6.1 - 30.6.4. DOI: 10.1109/IEDM.2016.7838515.

[27] Synopsys, Sentaurus ${ }^{\mathrm{TM}}$ Device User Guide K-2015.06, Jun. 2015.

[28] K. Ohmori, T. Matsuki, D. Ishikawa, T. Morooka, T. Aminaka, Y. Sugita, T. Chikyow, K. Shiraishi, Y. Nara, and K. Yamada, "Impact of additional factors in threshold voltage variability of metal/high-k gate stacks and its reduction by controlling crystalline structure and grain size in the metal gates," in Proc. IEEE Intern. Electron Devices Meeting $(I E D M)$, San Francisco, CA, USA, 2008, pp. 1-4. DOI: 10.1109/IEDM.2008.4796707.

[29] S. M. Nawaz and A. Mallik, "Effects of Device Scaling on the Performance of Junctionless FinFETs Due to Gate-Metal Work Function Variability and Random Dopant Fluctuations," IEEE Electron Device Letters, vol. 37, no. 8, pp. 958-961, Aug. 2016. DOI: 10.1109/LED.2016.2578349.

[30] E. Baravelli, M. Jurczak, N. Speciale, K. D. Meyer, and A. Dixit, "Impact of LER and Random Dopant Fluctuations on FinFET Matching Performance," IEEE Trans. Nanotechnology, vol. 7, no. 3, pp. 291-298, May 2008, 10.1109/TNANO2008.917838.

[31] International Technology Roadmap for Semiconductors (ITRS), 2013

[32] J.-P. Colinge, "Multiple-gate SOI MOSFETs," Solid-State Electronics, vol. 48, no. 6, pp. 897-905, Jun. 2004. DOI: 10.1016/j.sse.2003.12.020.

[33] Y. Li, H. T. Chang, C. N. Lai, P. J. Chao and C. Y. Chen, "Process variation effect, metal-gate work-function fluctuation and random dopant fluctuation of 10-nm gate-all-around silicon nanowire MOSFET devices," in Proc. IEEE Intern. Electron Devices Meeting (IEDM), Washington, DC, 2015, pp. 34.4.1-34.4.4.

[34] S. H. Chou, M. L. Fan and P. Su, "Investigation and Comparison of Work Function Variation for FinFET and UTB SOI Devices Using a Voronoi Approach," IEEE Trans. Electron Devices, vol. 60, no. 4, pp. 1485-1489, April 2013. DOi: 10.1109/TED.2013.2248087.

[35] J. Franco, L. Witters, A. Vandooren, H. Arimura, S. Sionke, V. Putcha, A. Vais, Q. Xie, M. Givens, F. Tang, X. Jiang, A. Subirats, A. Chasin, L.-Å. Ragnarsson, N. Horiguchi, B. Kaczer, D. Linten, and N. Collaert,
"Gate stack thermal stability and PBTI reliability challenges for 3D sequential integration: Demonstration of a suitable gate stack for top and bottom tier nMOS," in Proc. IEEE Int. Rel. Phys. Symp. (IRPS), Monterey, CA, 2017, pp. 2B-3.1-2B-3.5.

[36] T. Matsukawa, Y. Liu, W. Mizubayashi, J. Tsukada, H. Yamauchi, K. Endo, Y. Ishikawa, S. O'uchi, H. Ota, S. Migita, Y. Morita, and M. Masahara, "Suppressing Vt and Gm variability of FinFETs using amorphous metal gates for $14 \mathrm{~nm}$ and beyond," in Proc. IEEE Intern. Electron Devices Meeting (IEDM), San Francisco, CA, 2012, pp. 8.2.18.2.4.

[37] S. Medury, K. N. Bhat, and N. Bhat, "Threshold voltage modeling under size quantization for ultra-thin silicon double-gate metal-oxidesemiconductor field-effect transistor," Journ. Applied Physics, vol. 112, no. 2, Jul. 2012. DOI: 10.1063/1.4737779.

[38] N. Seoane, G. Indalecio, E. Comesaña, M. Aldegunde, A. J. GarcíaLoureiro and K. Kalna, "Random Dopant, Line-Edge Roughness, and Gate Workfunction Variability in a Nano InGaAs FinFET," IEEE Trans, Electron Devices, vol. 61, no. 2, pp. 466-472, Feb. 2014. DOI: 10.1109/TED.2013.2294213.

[39] D. Reid, C. Millar, S. Roy and A. Asenov, "Statistical Enhancement of the Evaluation of Combined RDD- and LER-Induced $\mathrm{V}_{\mathrm{T}}$ Variability: Lessons From $10^{5}$ Sample Simulations," IEEE Trans. Electron Devices, vol. 58, no. 8, pp. 2257-2265, Aug. 2011. DOI: 10.1109/TED.2011.2147317.

[40] L. Czornomaz, N. Daix, P. Kerber, K. Lister, D. Caimi, C. Rossel, M. Sousa, E. Uccelli, and J. Fompeyrine, "Scalability of ultra-thin-body and BOX InGaAs MOSFETs on silicon," in Proceedings of European SolidState Device Research Conference (ESSDERC), Bucharest, RO, 2013, pp. 143-146. DOI: 10.1109/ESSDERC.2013.6818839.

[41] S. Mittal, S. Gupta, A. Nainani, M. C. Abraham, K. Schuegraf, S. Lodha, and U. Ganguly, "Epitaxially Defined FinFET: Variability Resistant and High-Performance Technology," IEEE Trans. Electron Devices, vol. 61, no. 8, pp. 2711-2718, Aug. 2014. DOI: 10.1109/TED.2014.2329993.

Nicolò Zagni is a Ph.D. student at the University of Modena and Reggio Emilia. He received both BSc and MSc (summa cum laude) from the same institution. His work is focused on the characterization of III-V MOSFET devices, in particular regarding IV hysteresis and variability issues. In 2016, he attended the INC12 conference at imec, Belgium, as an invited young researcher from the European Commission.

Francesco Maria Puglisi (S'12, M'15) received the Ph.D. degree in Information and Communication Technology in 2015 from University of Modena and Reggio Emilia, Italy, where he is currently a Research Associate and Adjunct Professor. His activity focuses on the characterization, physical, and compact modeling of novel transistors and nonvolatile memories, especially RRAM, focusing on noise and variability.

Dr. Puglisi authored and coauthored more than 40 technical papers and serves in the Technical Program Committee of IEEE IRPS, IEEE IPFA, and ChinaRRAM conferences. He is the recipient of the Best Student Paper Award at the IEEE ICICDT 2013 Conference, 29-31 May 2013, Pavia, Italy.

Giovanni Verzellesi received the "Laurea" degree (summa cum laude) in Electrical Engineering from the University of Bologna, Bologna, Italy, in 1989, and the Ph.D. degree also in Electrical Engineering from the University of Padova, Padova, Italy, in 1994. In 1993-94 he was a visiting graduate student with the University of California, Santa Barbara (CA, USA).

From 1994 to 1999, he was with the University of Trento, Italy, as an Assistant Professor of Electronics. Since 1999, he has been with the University of Modena and Reggio Emilia, Italy, where he became Associate Professor in 2000 and Professor in 2006.

Paolo Pavan (SM'12) is Professor of Electronics at the University of Modena and Reggio Emilia, Italy, since 1994.

His research interests are in the characterization, modeling and optimization of nonvolatile memory devices and, more recently, in the development of safety critical and energy-aware applications for automotive electronics.

Prof. Pavan was in the IEDM Technical and Executive Committee from 2002 to 2006. He has been Guest Editor of the IEEE Transactions on Device and Material Reliability Special Issue on Nonvolatile Memories in Sept. 2004. He has been in the Technical Committee of VLSI-TSA from 2006 to 2010. He 
is in the Technical Committee of IRPS 2014; in ESSDERC and ESREF from 2012. He has been Technical Program Chair of ESSDERC 2014 and now in the Steering Board of ESSDERC. 